\title{
A Generalised Branch-and-Bound Approach and its Application in SAT Modulo Nonlinear Integer Arithmetic ${ }^{\star}$
}

\author{
Gereon Kremer, Florian Corzilius, and Erika Ábrahám \\ RWTH Aachen University, Germany
}

\begin{abstract}
The branch-and-bound framework has already been successfully applied in SAT-modulo-theories (SMT) solvers to check the satisfiability of linear integer arithmetic formulas. In this paper we study how it can be used in SMT solvers for non-linear integer arithmetic on top of two real-algebraic decision procedures: the virtual substitution and the cylindrical algebraic decomposition methods. We implemented this approach in our SMT solver SMT-RAT and compared it with the currently best performing SMT solvers for this logic, which are mostly based on bit-blasting. Furthermore, we implemented a combination of our approach with bit-blasting that outperforms the state-of-the-art SMT solvers for most instances.
\end{abstract}

\section{Introduction}

Satisfiability checking [7] aims to develop algorithms and tools to check the satisfiability of existentially quantified logical formulas. Driven by the success of SAT solving for propositional logic, fruitful initiatives were started to enrich propositional SAT solving with solver modules for different theories. SAT-modulotheories (SMT) solvers [6 make use of an efficient SAT solver to check the logical (Boolean) structure of formulas, and use different theory solver modules for checking the consistency of theory constraint sets in the underlying theory.

Besides theories, such as equality logic, uninterpreted functions, bit-vectors and arrays, SMT solvers also support arithmetic theories. Apart from interval constraint propagation (ICP), SMT solving for quantifier-free linear real arithmetic ( $\left.Q F_{-} L R A\right)$ often makes use of linear programming techniques such as the simplex method [15]. For quantifier-free non-linear real arithmetic $\left(Q F_{-} N R A\right)$, algebraic decision procedures, e.g. the virtual substitution (VS) method 28] or the cylindrical algebraic decomposition method $(C A D)$ [12] can be used.

There are several powerful SMT solvers, e.g., CVC4 4], MathSAT5 [1], Sateen [24], veriT [9], Yices2 [18] and Z3 [26] which offer solutions for quantifier-free linear integer arithmetic ( $\left.Q F_{-} L I A\right)$ problems that mostly build on the ideas in

\footnotetext{
* The final authenticated version is available online at https://doi.org/10.1007/978-3319-45641-6_21.
} 
17. Closely related to our work is the SMT-adaptation of the branch-and-bound $(B B)$ framework [27 in MathSAT5.

When moving from the real to the integer domain, non-linear arithmetic becomes undecidable. Despite this fact, there are a few SMT solvers that support the satisfiability check of quantifier-free non-linear integer arithmetic (QF_NIA) formulas, either for restricted domains (in which case the domain becomes finite and the problem becomes decidable) or in an incomplete manner. Some of these SMT solvers apply linearisation [8, whereas other tools, such as iSAT3 [19] and raSAT [23, use interval constraint propagation adapted to the integer domain. To the best of our knowledge, all other prominent solvers, such as AProVE [20], CVC4 or Z3, apply mainly bit-blasting, which exploits SAT solvers by the use of a binary encoding of bounded integer domains.

Although the satisfiability problem for QF_NIA is undecidable, we see in the employment and adaptation of algebraic decision procedures a promising alternative to achieve incomplete but practically efficient satisfiability checking solutions for QF_NIA problems. Such solutions are urgently needed in several research areas to open new possibilities and enable novel approaches. A typical example is the field of program verification where, for instance, deductive proof systems often generate QF_NIA formulas as verification conditions (see e.g. [3]). Just to mention a second example, for the termination analysis of programs often QF_NIA termination conditions need to be checked for satisfiability [8]. Currently, non-linear integer arithmetic problems appearing in these areas are often solved using, e.g., theorem proving, linearisation or bit-blasting.

Today's SMT solvers neither exploit adaptations of algebraic QF_NRA decision procedures for finding QF_NIA solutions ${ }^{1}$ nor use the BB framework to check QF_NIA formulas for satisfiability. In this paper we investigate these issues. The main contributions of this paper are:

- We show on the example of the CAD method that some algebraic decision procedures for QF_NRA can be adapted to drive their search towards integer solutions. Experimental results show that this approach works surprisingly well on the satisfiable problem instances which we considered.

- We propose improvements for the general integration of BB in SMT solving.

- We show on the examples of the VS and the CAD methods, how algebraic QF_NRA decision procedures can be embedded into the BB framework to solve QF_NIA problems.

- Finally, we provide experimental results to illustrate how different decision procedures can be strategically adapted, combined and embedded in the BB framework to tackle the challenge of QF_NIA satisfiability checking.

The rest of the paper is structured as follows. We start in Section 2 with preliminaries on QF_NIA, SMT solving, and the VS and the CAD methods. In

\footnotetext{
${ }^{1} \mathrm{Z} 3$ has a command-line option to solve, instead of QF_NIA problems, their QF_NRA relaxations. This way $\mathrm{Z3}$ can detect unsatisfiability (no real solution is found) or sometimes even satisfiability (the found real solution happens to be integer), but otherwise it returns "unknown".
} 
Section 3 we present a general framework for BB. Section 4 and 5 are devoted to the integration of the VS and the CAD methods, respectively, into the BB framework. After an experimental evaluation of the presented approach in Section 7 . we conclude the paper in Section 8 .

\section{Preliminaries}

(Quantifier-free non-linear arithmetic) formulas $\varphi$ are Boolean combinations of constraints $c$ which compare polynomials $p$ to 0 . A polynomial $p$ can be a constant, a variable $x$, or a sum, difference or product of polynomials:

$$
\begin{array}{cl|c|c|c|c|c}
p & ::=0 & 1 & x & (p+p) & (p-p) & (p \cdot p) \\
c & ::=p=0 & p<0 & & & \\
\varphi & :=c & \mid \neg \varphi) & \mid & (\varphi \wedge \varphi) & &
\end{array}
$$

We use further operators such as disjunction $\vee$, implication $\Rightarrow$ and comparisons $>, \leq, \geq, \neq$, which are defined as syntactic sugar the standard way, and standard syntactic simplifications (e.g., we omit parentheses based on the standard operator binding order, write $p_{1} p_{2}$ instead of $p_{1} \cdot p_{2},-p$ instead of $\left.0-p\right)$.

We use $\mathbb{Z}\left[x_{1}, \ldots, x_{n}\right]$ to denote the set of all polynomials (with integer coefficients) over the variables $x_{1}, \ldots, x_{n}$ for some $n \geq 1$. A polynomial $p \in$ $\mathbb{Z}\left[x_{1}, \ldots, x_{n}\right]$ is called univariate if $n=1$, and multivariate otherwise. By $\operatorname{Var}(\varphi)$, $\operatorname{Pol}(\varphi)$ and $\operatorname{Con}(\varphi)$ we refer to the set of all variables, polynomials and constraints occurring in the formula $\varphi$, respectively; especially, $\operatorname{Pol}(p \sim 0)$ denotes the polynomial $p$ of a given constraint $p \sim 0$ with $\sim \in\{<, \leq,=, \neq, \geq,>\}$.

Each polynomial $p \in \mathbb{Z}\left[x_{1}, \ldots, x_{n}\right]$ can be equivalently transformed to the form $a_{k} x_{1}^{e_{1, k}} \ldots x_{n}^{e_{n, k}}+\ldots+a_{1} x_{1}^{e_{1,1}} \ldots x_{n}^{e_{n, 1}}+a_{0}$ with coefficients $a_{j} \in \mathbb{Z}$ for $0 \leq i \leq n$ and exponents $e_{i, j} \in \mathbb{N}_{0}$ for $1 \leq i \leq n$ and $1 \leq j \leq k$. We call $m_{j}:=x_{1}^{e_{1, j}} \ldots x_{n}^{e_{n, j}}$ a monomial, $t_{j}:=a_{j} m_{j}$ a term, and $a_{0}$ the constant part of $p$. In the following, we assume that polynomials are in the above form with pairwise different monomials; note that this form is unique up to the ordering of the terms. By $p_{1} \equiv p_{2}\left(\varphi_{1} \equiv \varphi_{2}\right)$ we denote that the polynomials $p_{1}$ and $p_{2}$ (the formulas $\varphi_{1}$ and $\varphi_{2}$ ) can be transformed to the same form. By $\operatorname{deg}\left(t_{j}\right):=\sum_{i=1}^{n} e_{i, j}$ we denote the degree of the term $t_{j}$. By $\operatorname{deg}(p):=\max _{1 \leq j \leq k} \operatorname{deg}\left(t_{j}\right)$ we denote the degree of $p$ and by $\operatorname{deg}\left(x_{i}, p\right):=\max _{1 \leq j \leq k} e_{i, j}$ the degree of $x_{i}$ in $p$. A polynomial $p$ is linear, if $\operatorname{deg}(p) \leq 1$, and non-linear otherwise. A formula $\varphi$ is linear, if all polynomials $p \in \operatorname{Pol}(\varphi)$ are linear, and non-linear otherwise.

We use the standard semantics of arithmetic formulas. In the theory of quantifier-free non-linear integer arithmetic ( $\left.Q F_{-} N I A\right)$, all variables $x_{i}$ are integervalued (the domain of $x_{i}$, denoted by $\operatorname{Dom}\left(x_{i}\right)$, is $\mathbb{Z}$ ); in quantifier-free non-linear real arithmetic $\left(Q F_{-} N R A\right)$ all variables $x_{i}$ are real-valued $\left(\operatorname{Dom}\left(x_{i}\right)=\mathbb{R}\right)$; in the theory of non-linear mixed integer-real arithmetic $\left(Q F_{-} N I R A\right)$, variables can have either domain. We denote by $\varphi_{\mathbb{Z}}\left(\varphi_{\mathbb{R}}\right)$ that $\varphi$ is interpreted as a QF_NIA (QF_NRA) formula, and call $\varphi_{\mathbb{R}}$ the real relaxation of $\varphi_{\mathbb{Z}}$.

As a preprocessing step for QF_NIA formulas, we replace inequalities $p \neq 0$ by $p<0 \vee p>0$. Furthermore, based on the integrality of all variables, we simplify 


\begin{tabular}{r|rrrrr} 
& $<$ & $\leq$ & $=$ & $\geq$ & $>$ \\
\hline$a_{0}^{\prime} \in \mathbb{Z}$ & $r+a_{0}^{\prime}+1 \leq 0$ & $r+a_{0}^{\prime} \leq 0$ & $r+a_{0}^{\prime}=0$ & $r+a_{0}^{\prime} \geq 0$ & $r+a_{0}^{\prime}-1 \geq 0$ \\
$a_{0}^{\prime} \notin \mathbb{Z}$ & $r+\left\lceil a_{0}^{\prime}\right\rceil \leq 0$ & $r+\left\lceil a_{0}^{\prime}\right\rceil \leq 0$ & false & $r+\left\lfloor a_{0}^{\prime}\right\rfloor \geq 0$ & $r+\left\lfloor a_{0}^{\prime}\right\rfloor \geq 0$
\end{tabular}

Table 1: Simplification of a QF_NIA constraint $\left(\sum_{i=1}^{k} a_{i} m_{i}\right)+a_{0} \sim 0$, where $g$ is the greatest common divisor of $a_{1}, \ldots, a_{k}, a_{0}^{\prime}:=\frac{a_{0}}{g}$ and $r:=\sum_{i=1}^{k} \frac{a_{i}}{g} m_{i}$

constraints in the formula according to Table 1 . After these simplifications, only the relations $=, \geq$ and $\leq$ (but no $<,>$ nor $\neq$ ) appear in the formulas.

The substitution of a variable $x$ by a value $v \in \operatorname{Dom}(x)$ in a formula $\varphi$ is denoted by $\varphi[v / x]$. A value $\left(v_{1}, \ldots, v_{n}\right) \in \mathbb{R}^{n}$ is a real root or zero of a polynomial $p \in \mathbb{Z}\left[x_{1}, \ldots, x_{n}\right]$ if $p\left(v_{1}, \ldots, v_{n}\right):=p\left[v_{1} / x_{1}\right] \ldots\left[v_{n} / x_{n}\right] \equiv 0$. A value $\left(v_{1}, \ldots, v_{n}\right) \in \mathbb{R}^{n}$ is a solution of a formula $\varphi$ with $\operatorname{Var}(\varphi)=\left\{x_{1}, \ldots, x_{n}\right\}$ if $\varphi\left(v_{1}, \ldots, v_{n}\right):=\varphi\left[v_{1} / x_{1}\right] \ldots\left[v_{n} / x_{n}\right] \equiv$ true.

The satisfiability checking problem is the problem to decide whether there exists a solution for a given formula $\varphi$. Note that checking the satisfiability of a quantifier-free formula $\varphi$ with $\operatorname{Var}(\varphi)=\left\{x_{1}, \ldots, x_{n}\right\}$ and checking the validity of the existentially quantified formula $\exists x_{1} \ldots \exists x_{n} . \varphi$ define the same problem.

\section{$2.1 \quad$ SAT-modulo-theories solving}

For the satisfiability check of logical formulas over some theories, SAT-modulotheories (SMT) solvers combine a SAT solver with one or more theory solvers. The SAT solver is used for the efficient exploration of the logical structure of the input formula, whereas the theory solver(s), implementing some decision procedures for the underlying theory, are used to check the consistency of sets (conjunctions) of certain theory constraints. The SMT-solving framework is illustrated in Figure 1 .

In this work we consider a lesslazy SMT-solving approach based on

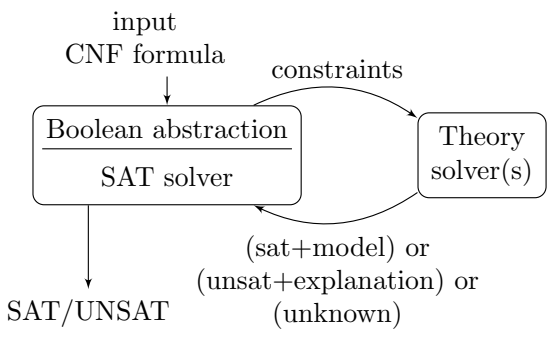

Fig. 1: The SMT solving framework DPLL 16] SAT solving. The input formula is brought to negation normal form, negation is applied to atomic constraints, and finally conjunctive normal form (CNF) is built (using Tseitin's transformation), resulting in a conjunction of disjunctions of atomic (not negated) constraints. The SAT solver tries to find a satisfying solution for the Boolean skeleton of the input formula, which is the propositional logic formula obtained by replacing each constraint $c$ by a fresh proposition $h_{c}$. In other words, the SAT solver tries to determine a set of constraints such that the input formula is satisfiable if the determined constraints have a common 
solution. During its search, the SAT solver works "less lazily", i.e., it asks the theory solver(s) regularly whether the set of those constraints, whose abstraction variables are true in the current partial assignment, are together consistent. Note that we do not need to pass the negation of constraints with false abstraction variables to the theory solver, because all abstraction variables appear in the transformed formula without negation. Therefore, all clauses are still satisfiable even if we wrongfully assign a variable to false.

The DPLL algorithm, used in most state-of-the-art SAT solvers, executes the following loop: It repeatedly makes a decision, assuming a certain value for some proposition. Then it applies Boolean constraint propagation $(B C P)$, thereby identifying variable assignments that are implied by the last decision. If the propagation succeeds without conflicts, a new decision is made. However, the propagation might also lead to a conflict, which means that the current partial assignment cannot be extended to a full satisfying solution. In the latter case conflict resolution is applied to determine which decisions led to the conflict. After backtracking, i.e., undoing some of the previous decisions, the SAT solver learns a new clause to exclude the found conflict (and other similar ones) from the future search and continues the search in other parts of the search space.

In the less-lazy SMT-solving context, theory solvers need to be able to work incrementally, i.e., to extend a previously received set of constraints with new constraints and to check the extended set for consistency while reusing collected information from the last check in order to improve the performance; similarly, they need to be able to backtrack, i.e., to remove constraints from their current constraint sets. Furthermore, to enable the SAT solver to resolve theory-rooted conflicts, the theory solver has to return an explanation if it detects inconsistency, usually in form of an inconsistent subset of its received constraints.

We use our SMT-solving toolbox SMT-RAT [14 to implement the approaches described in this work, and to compare the results to other approaches. SMT-RAT provides a rich set of SMT-compliant implementations of QF_NRA/ QF_NIA procedures. These procedures are encapsulated in modules, which are executed by our SMT solver according to a user-defined strategy. In this work, we will use only sequential strategies, where a preprocessing module, a SAT solver module, and one or more theory solver modules are arranged sequentially. The SAT solver sends theory constraints in an incremental fashion to the first theory solver module, which tries to determine whether its received constraints have a common satisfying solution. The first theory solver might also pass on sub-problem: $2^{2}$ to be checked for satisfiability to the next theory solver module and so on. Each theory solver module returns to its caller module (i) either satisfiability along with a model if requested, (ii) or unsatisfiability and an explanation in form of an infeasible subset of its received formulas, (iii) or unknown. Besides modules for parsing the input problem and transforming it to conjunctive normal form as requested by SAT solving, we use a MiniSat-based SAT solver module and

\footnotetext{
${ }^{2}$ These sub-problems are not necessarily constraints or conjunctions of constraints, but in general formulas with arbitrary Boolean structure.
} 
theory solver modules implementing the simplex, the VS and the CAD methods, and a theory solver module for bit-blasting.

\subsection{Virtual substitution}

The virtual substitution (VS) method 28] is an incomplete decision procedure for non-linear real arithmetic. As we aim at satisfiability checking, we restrict ourselves to the quantifier-free fragment QF_NRA (where we understand all free variables as existentially quantified). Given a QF_NRA formula $\varphi_{\mathbb{R}}$ in variables $\operatorname{Var}\left(\varphi_{\mathbb{R}}\right)=\left\{x_{1}, \ldots, x_{n}\right\}$, the VS iteratively eliminates variables that appear at most quadratic in $\varphi_{\mathbb{R}}$. Assume w.l.o.g. that we want to eliminate a variable $x_{n}$, such that $\operatorname{deg}\left(x_{n}, p\right) \leq 2$ for all $p \in \operatorname{Pol}\left(\varphi_{\mathbb{R}}\right)$.

In the univariate case, i.e. $\operatorname{Pol}\left(\varphi_{\mathbb{R}}\right) \subseteq \mathbb{Z}\left[x_{n}\right]$, we can use the solution equation for quadratic polynomials to determine the real roots of all $p \in \operatorname{Pol}\left(\varphi_{\mathbb{R}}\right)$. They separate regions in which each $p \in \operatorname{Pol}\left(\varphi_{\mathbb{R}}\right)$ is sign-invariant. We determine for each of those sign-invariant regions a representative element, which we collect in the set of test candidates $T\left(x_{n}, \varphi_{\mathbb{R}}\right)$. Due to the sign-invariance of those regions, $\varphi_{\mathbb{R}}$ is satisfiable if and only if one of the test candidates satisfies the formula.

Even if the formula contains multivariate polynomials, we can apply the solution equation to determine real roots $t_{i}$ and the side conditions $S C\left(t_{i}\right)$ for their existence (radicand non-negative, denominator is not zero), if we interpret multivariate polynomials $p \in \mathbb{Z}\left[x_{1}, \ldots, x_{n}\right]$ as univariate polynomials with polynomial coefficients $p \in \mathbb{Z}\left[x_{1}, \ldots, x_{n-1}\right]\left[x_{n}\right]$. However, now the results are parametric in $x_{1}, \ldots, x_{n-1}$, therefore we know neither the existence nor the order of the roots; here, the VS uses $-\infty$ as a test candidate from the left-most signinvariant region, and infinitesimals $\epsilon$ in order to represent with $t+\epsilon$ the sign-invariant region on the right of $t$. As the test candidates might contain fractions, radicals, $-\infty$ and $\epsilon$, standard substitution $\left[t_{i} / x_{n}\right]$ can lead to improper expressions which are not arithmetic formulas. Instead we use the virtual substitution $\left[t_{i} / / x_{n}\right]$ which resolves these improper expressions with special rules 28 resulting in a QF_NRA formula $\varphi_{\mathbb{R}}\left[t_{i} / / x_{n}\right]$ that is satisfiability-equivalent to
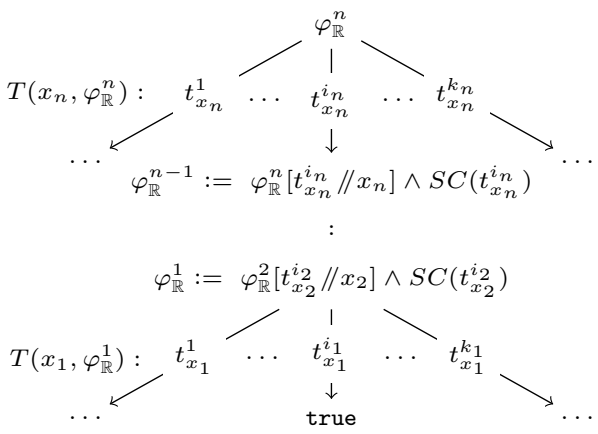

Fig. 2: Possible VS depth-first search $\varphi_{\mathbb{R}}\left[t_{i} / x_{n}\right]$.

In summary, the VS specifies a finite set $T\left(x_{n}, \varphi_{\mathbb{R}}\right)$ of (symbolic) test candidates (TCs) for $x_{n}$ in $\varphi_{\mathbb{R}}$, and for each TC $t \in T\left(x_{n}, \varphi_{\mathbb{R}}\right)$ some side conditions $S C(t)$, such that

$$
\varphi_{\mathbb{R}} \text { is satisfiable } \Leftrightarrow \bigvee_{t \in T\left(x_{n}, \varphi_{\mathbb{R}}\right)}\left(\varphi_{\mathbb{R}}\left[t / / x_{n}\right] \wedge S C(t)\right) \text { is satisfiable . }
$$




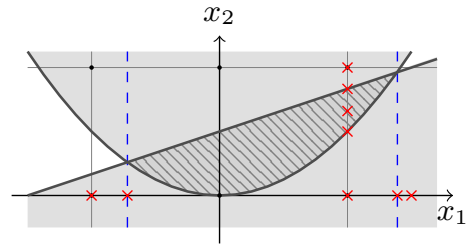

(a) Plot of the solution space and the sample points

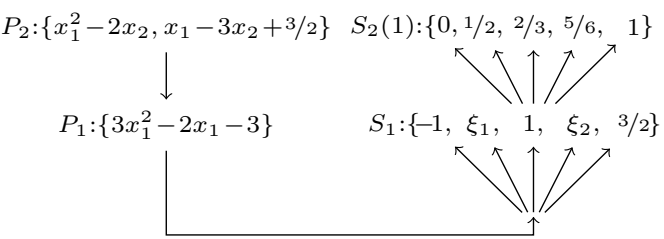

(b) Schematic overview of the CAD method

Fig. 3: CAD example for $c_{1}: x_{1}^{2}-2 x_{2} \leq 0$ and $c_{2}: x_{1}-3 x_{2}+3 / 2 \geq 0$

In [13] we presented an implementation of the VS for satisfiability checking, which executes a depth-first search for a true leaf as illustrated in Figure 2, a solution can be read off the solution path from the root to the true leaf.

\subsection{Cylindrical algebraic decomposition}

The cylindrical algebraic decomposition $(C A D)$ 1212 is a complete decision procedure for non-linear real arithmetic, which we will use in the SMT solving context for checking the satisfiability of QF_NRA formulas. Due to space restriction, we give only a high-level description here.

Given a formula $\varphi_{\mathbb{R}}$ in variables $x_{1}, \ldots, x_{n}$, the CAD method partitions $\mathbb{R}^{n}$ into a finite number of disjoint $n$-dimensional cells. Each of them is a connected semi-algebraic set over which all polynomials in $\operatorname{Pol}\left(\varphi_{\mathbb{R}}\right)$ are sign-invariant and thus either all or none of the points in a cell satisfy $\varphi_{\mathbb{R}}$. The cells are constructed to be cylindrical: the projections of any two $n$-dimensional cells onto the $k$ dimensional space $(1 \leq k<n)$ are either identical or disjoint. The $n$-dimensional cells with identical $k$-dimensional projections $S$ form cylinders $S \times \mathbb{R}^{n-k}$.

The CAD method uses a two-phase approach to compute such a partitioning. In the projection phase, a projection operator is applied to the input set $P_{n}=$ $\operatorname{Pol}\left(\varphi_{\mathbb{R}}\right)$ of $n$-dimensional polynomials with respect to the variable $x_{n}$, yielding a set $P_{n-1} \subset \mathbb{Z}\left[x_{1}, \ldots, x_{n-1}\right]$ of $(n-1)$-dimensional polynomials, whose real roots constitute the boundaries of the $(n-1)$-dimensional projections of the $n$-dimensional CAD cells. The projection operator is applied recursively until a set $P_{1} \subset \mathbb{Z}\left[x_{1}\right]$ of univariate polynomials is obtained. Note that this imposes a fixed variable ordering that cannot easily be changed without recomputing the projection. Much work has been done on providing efficient methods for computing preferably small projections, for example in [1022 25].

In the second phase called lifting or construction, the CAD method constructs a sample point for each of the cells. It first isolates the real roots $\xi_{1}, \ldots, \xi_{k}$ of the polynomials in $P_{1}$ which constitute the boundaries of the 1-dimensional projections of the cells which are the intervals $I=\left\{\left(-\infty, \xi_{1}\right),\left[\xi_{1}, \xi_{1}\right],\left(\xi_{1}, \xi_{2}\right), \ldots,\left(\xi_{k}, \infty\right)\right\}$. We choose a sample point from each of these intervals $I_{i}$, resulting in a sample set $S_{1}=\left\{s_{1}, \ldots, s_{2 k+1}\right\}$. Each sample point $s \in S_{1}$ from some interval $I_{i}$ is now lifted 
using the polynomials from $P_{2}$ : each polynomial from $P_{2}$ is partially evaluated on $s$ which results in a set of univariate polynomials whose real roots $\left\{\xi_{1}^{s}, \ldots \xi_{l_{s}}^{s}\right\}$ are again isolated. For each real root $\left\{\xi_{1}^{s}, \ldots \xi_{l_{s}}^{s}\right\}$ there exists a surface $I_{i} \times \xi_{j}^{s}$ and together these surfaces separate the cylinder $I_{i} \times \mathbb{R}$ into $2 \cdot l_{s}+1$ individual cells. Assuming that $\xi_{1}^{s}<\ldots<\xi_{l_{s}}^{s}$, these cells are the surfaces $I_{i} \times \xi_{j}^{s}\left(\right.$ for $\left.j=1, \ldots, l_{s}\right)$, the regions between two surfaces $I_{i} \times\left(\xi_{j}^{s}, \xi_{j+1}^{s}\right)$ (for $j=1, \ldots, l_{s}-1$ ), and the regions below and above all separating surfaces $I_{i} \times\left(-\infty, \xi_{1}^{s}\right)$ and $I_{i} \times\left(\xi_{l_{s}}^{s}, \infty\right)$. We can again take sample points from these cells and repeat the lifting procedure until we obtain $n$-dimensional sample points that are representatives for the $n$-dimensional cells that are sign-invariant with respect to the polynomials in $P_{n}$. We evaluate $\varphi_{\mathbb{R}}$ for each $n$-dimensional sample point to check whether one of them satisfies the formula, in which case we obtain a satisfying solution, otherwise the formula is not satisfiable.

The CAD method is illustrated on a 2-dimensional example in Figure 3 . Figure $3 \mathrm{a}$ depicts the solution space, while Figure $3 \mathrm{~b}$ visualises the CAD computation. The sample point $(1,2 / 3)$ satisfies the formula $x_{1}^{2}-2 x \leq 0 \wedge x_{1}-3 x_{2}+3 / 2 \geq 0$. Note that we can choose a sample point from intervals between real roots. This choice is important when searching for integer solutions: while there is no integer solution for the sample point 1 from the interval $\left(\xi_{1}, \xi_{2}\right)$, selecting 0 instead of 1 would have resulted in the integer solution $(0,0)$.

\section{A General Branch-and-Bound Framework}

A popular approach to check QF LIA formulas $\varphi_{\mathbb{Z}}$ for satisfiability is the branchand-bound $(B B)$ framework [27. It first considers the relaxed problem $\varphi_{\mathbb{R}}$ in the real domain. If the relaxed problem is unsatisfiable then the integer problem is unsatisfiable, too. Otherwise, if there exists a real solution then it is either integer-valued, in which case $\varphi_{\mathbb{Z}}$ is satisfiable, or it contains a non-integer value $r \in \mathbb{R} \backslash \mathbb{Z}$ for an integer-valued variable $x$. In the latter case a branching takes place: $\mathrm{BB}$ reduces the relaxed solution space by excluding all values between $\lfloor r\rfloor=\max \left\{r^{\prime} \in \mathbb{Z} \mid r^{\prime} \leq r\right\}$ and $\lceil r\rceil=\min \left\{r^{\prime} \in \mathbb{Z} \mid r^{\prime} \geq r\right\}$ in the $x$-dimension, described by the formula $\varphi^{\prime}=\varphi \wedge(x \leq\lfloor r\rfloor \vee x \geq\lceil r\rceil)$. This procedure is applied iteratively, i.e., $\mathrm{BB}$ will now search for real-valued solutions for $\varphi^{\prime}$. BB terminates if either an integer solution is found or the relaxation is unsatisfiable. Note that $\mathrm{BB}$ is in general incomplete even for the decidable logic QF_LIA.

The most well-known application combines BB with the simplex method. As branching introduces disjunctions and thus in general non-convexity, branching is implemented by case splitting: in one search branch we assume $x \leq\lfloor r\rfloor$, and in a second search branch we assume $x \geq\lceil r\rceil$. Depending on the heuristics, the search can be depth-first (full check of one of the branches, before the other branch is considered), breadth-first (check real relaxations in all current open branches before further branching is applied), or it can follow a more sophisticated strategy.

The combination of $\mathrm{BB}$ with the simplex method was also explored in the SMT-solving context [17. The advantage in this setting is that we have more possibilities to design the branching. 
- We can integrate a theory solver module based on the simplex method as described above, implementing BB internally in the theory solver by case splitting. It comes with the advantage that case splitting is always local to the current problem of the theory solver and does not affect later problems, and with the disadvantage that we cannot exploit the advantages of learning, i.e., to remember reasons of unsatisfiability in certain branches and use this information to speed up the search in other branches.

- Alternatively, given a non-integer solution $r$ for a variable $x$ found by the theory solver on a relaxed problem, we can lift the branching to the SAT solver by extending the current formula with a new clause $(x \leq\lfloor r\rfloor \vee x \geq\lceil r\rceil)$ [5. The newly added clause must be satisfied in order to satisfy the extended formula. Therefore, the SAT solver assigns (the Boolean abstraction variable of) either $x \leq\lfloor r\rfloor$ or $x \geq\lceil r\rceil$ to true, i.e., the branching takes place. On the positive side, lifting branching information and branching decisions to the SAT solver allows us to learn from information collected in one branch, and to use this learnt information to speed up the search in other branches. On the negative side, the branching is not local anymore as it is remembered in a learnt clause. Therefore, it might cause unwanted splittings in later searches.

To unify advantages, MathSAT5 21 implements a combined approach with theory-internal splitting up to a given depth and splitting at the logical level beyond this threshold.

Following the $\mathrm{BB}$ approach in combination with the simplex method, we can transfer the idea also to non-linear integer arithmetic: We can use QF_NRA decision procedures to find solutions for the relaxed problem and branch at noninteger solutions of integer-valued variables. However, there are some important differences. Most notably, the computational effort for checking the satisfiability of non-linear real-arithmetic problems is much higher than in the linear case. If we have found a real-valued solution and apply branching to find integer solutions, the branching will refine the search in the VS and CAD methods: it will create additional test candidates for the VS and new sample points for the CAD method, which will serve as roots for new sub-trees in the search tree. However, the search trees in both branches have a lot in common, that means, a lot of the same work has to be done for both sides of the branches. To prevent the solvers from doing much unnecessary work, we has to carefully design the BB procedure.

- Branching has to be lifted to the SAT solver level to enable learning, both in the form of branching lemmas as well as explanations for unsatisfiability in different branches.

- Learning explanations will allow us to speed up the search by transferring useful information between different branches. However, we need to handle branching lemmas thoughtfully and assure that learnt branching lemmas will not lead to branching for all future sub-problems, but only for "similar" ones where the branching will probably be useful.

- As branching refines the search, it has to work in an incremental fashion without resetting solver states. 


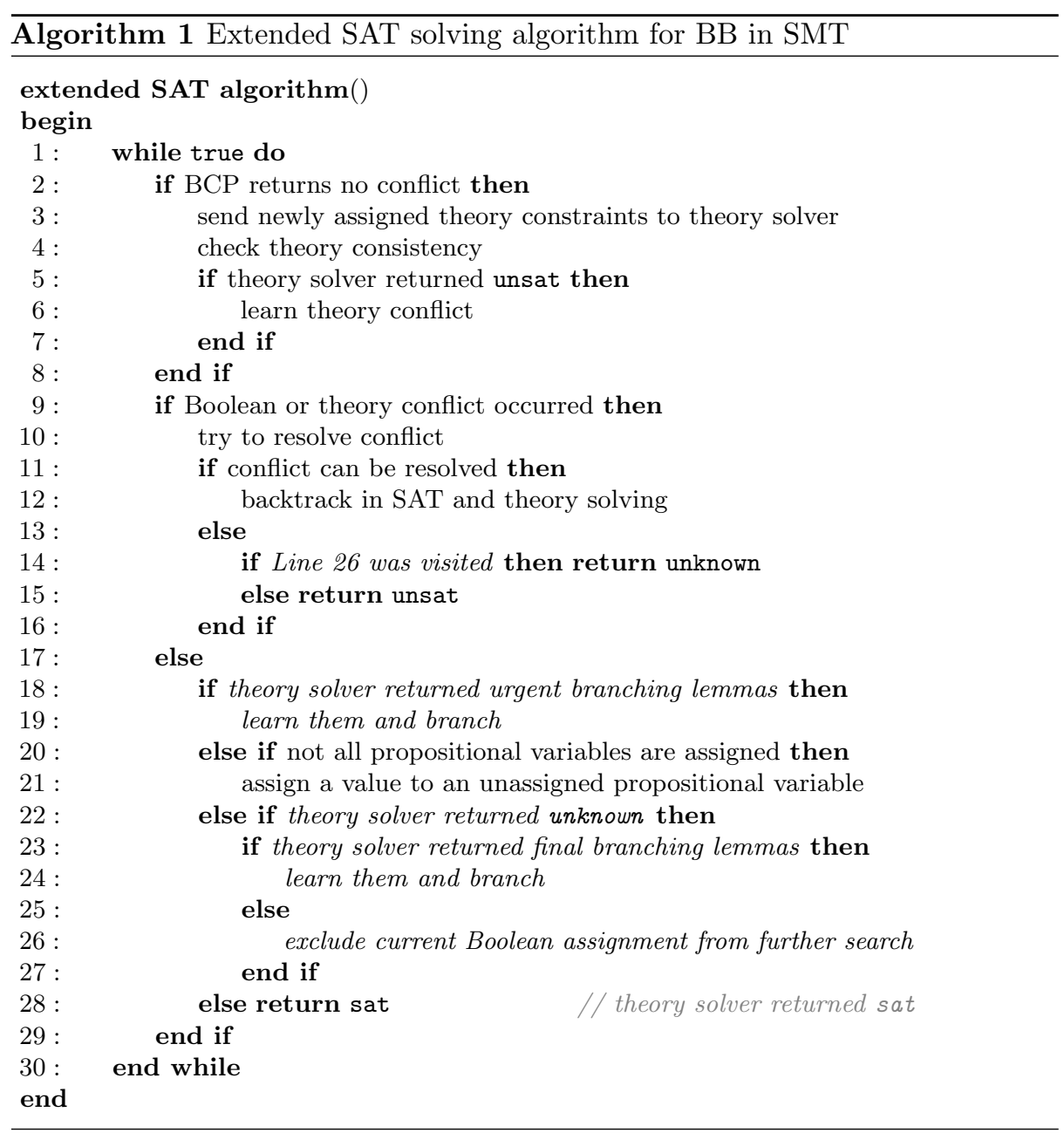

- If possible, the search strategies of the underlying QF_NRA decision procedures have to be tuned to prefer integer solutions (and if they can choose between different integer values, they must choose the most "promising" one).

- Last but not least, as the performance of solving QF_NRA formulas for satisfiability highly improves if different theory solvers implementing different decision procedures are used in combination, a practically relevant BB approach for QF_NIA should support this option.

\subsection{Processing branching lemmas in DPLL(T) SAT solving}

As mentioned before, an SMT solver combines a SAT solver and one or more theory solver modules. First we discuss our general approach of adapting these modules to implement BB for non-linear arithmetic, as described in Algorithm 1 . 
If we remove the Lines 14, 18, $19,22,27$ from Algorithm 1 (printed in italic font) then we achieve the standard SMT framework without $\mathrm{BB}$ embedding. This basic algorithm first applies Boolean constraint propagation (BCP, Line 2 ) to detect implications of current decisions. If BCP does not lead to a conflict, the consistency in the theory domain is checked (Lines 34 4 . If the theory constraints, which have to hold according to the current Boolean assignment, are inconsistent (Line 5) then the theory solver provides an explanation (an infeasible subset of its input constraints). We negate this explanation (resulting in a tautology) and add its Boolean abstraction as a new clause to the clause set to exclude this theory conflict from future search (Line 6); If either the BCP led to a Boolean conflict or a theory conflict occurred, then the solver tries to resolve the conflict (Line 10). If the conflict can be resolved (Line 11), then backtracking removes some of the decisions that led to the conflict; note that also the corresponding theory constraints will be removed from the input constraint list of the underlying theory solver (Line 12. As a result of a successful conflict resolution, a new clause will be learnt that will cause new implications in the next BCP iteration. Otherwise, if the conflict cannot be resolved, the input formula is unsatisfiable (Line 15).

Otherwise, if no conflict occurred, either all variables are assigned, in which case we have found a full solution (Line 28), or we choose one unassigned variable to which we assign a certain value (Lines 20 21) and propagate this when executing the next iteration of the main loop.

We modify this algorithm as follows. Firstly, additionally to sat and unsat, we allow theory solvers to return unknown. We do so because it is possible that the underlying theory solving procedure cannot determine the consistency of the set of constraints at hand. For instance, since QF_NIA is undecidable, a theory solver for QF_NIA can only be incomplete. This means either that the theory solver has non-terminating cases (which none of our theory solvers do), or that the theory solver relaxes each problem to a version that is decidable (e.g. QF_NRA). The latter case is only possible if we allow inconclusive answers, in which case the theory solver will return unknown. If the Boolean assignment is partial and the theory solver returns unknown, the SAT solver continues its search. If a full satisfying Boolean assignment was found by the SAT solver, but the theory solver cannot determine whether the solution is consistent in the (integer) theory, then the SAT solver excludes the current Boolean assignment from further search (by learning a clause in Line 26) and continues its search; if the following search detects a satisfying solution then the SMT solver returns sat, otherwise it returns unknown (Line 14).

Additionally to returning unknown, a theory solver can also return a so-called lemma that explains the inconclusive answer in more detail and that helps the SMT solver in finding a conclusive answer. This can happen for example if the theory solver has found a solution for the real relaxation of its input problem $\varphi$, but it is not integer-valued. In this case, the theory solver might return a branching lemma of the form

$$
\left(c_{1} \wedge \ldots \wedge c_{k}\right) \Rightarrow(x \leq\lfloor r\rfloor \vee x \geq\lceil r\rceil)
$$


demanding to split the domain of the integer-valued variable $x$ at the non-integer value $r \in \mathbb{R} \backslash \mathbb{Z}$, under the condition that the branching premise $c_{1} \wedge \ldots \wedge c_{k}$ with $\left\{c_{1}, \ldots, c_{k}\right\} \subseteq \operatorname{Con}(\varphi)$ holds. Additionally, the theory solver can specify which of the two branches it prefers to start with. We call the Boolean abstraction $\left(\neg h_{c_{1}} \vee \ldots \vee \neg h_{c_{k}} \vee h_{x \leq\lfloor r\rfloor} \vee h_{x \geq\lceil r\rceil}\right)$ of the branching lemma in Eq. 2 a branching clause and its last two (possibly fresh) literals branching literals.

Branching lemmas can be either urgent or fina ${ }^{3}$. Urgent branching lemmas are immediately abstracted, added to the SAT solver's clause set and used for branching (Lines 18 19). Final branching lemmas are relevant only if the SAT solver has a full satisfying Boolean assignment (Lines 23 24). When a branching clause is added, one of its branching literals (the one that was not preferred by the theory solver) will be assigned false (thus, if the branching premise is true, BCP will assign true to the preferred branching literal; this way we prevent that both branching literals become true, what would result in a theory conflict). Afterwards, we handle the branching clause just as any learnt clause and benefit from the usual reasoning and learning ${ }^{4}$ process, which yields the best performance according to our experience.

To prevent unnecessary branchings, we assign always the value false to branching literals as decision variables in Line 29 . Remember that only constraints with true abstraction variables will be passed to the theory solver. I.e., only branching clauses whose premise is true play a role in the theory, and for those clauses only one of the branching literals.

\section{Branch-and-Bound with Virtual Substitution}

In this section we present how the VS method as introduced in Section 2.2 can be embedded into the BB framework to check the satisfiability of a given QF_NIA formula $\varphi_{\mathbb{Z}}^{n}$ over (theory) variables $x_{1}, \ldots, x_{n}$. First we apply VS on the real relaxation $\varphi_{\mathbb{R}}^{n}$ of $\varphi_{\mathbb{Z}}^{n}$. If we determine unsatisfiability, we know that $\varphi_{\mathbb{Z}}^{n}$ is also unsatisfiable. Otherwise, if we have found a solution $S$ with the VS for $\varphi_{\mathbb{R}}^{n}$, as illustrated in Figure 2, then $S$ maps the variables $\operatorname{Var}\left(\varphi_{\mathbb{R}}^{n}\right)=\left\{x_{1}, \ldots, x_{n}\right\}$ to TCs $S\left(x_{j}\right)=t_{x_{j}}^{i_{j}}(1 \leq j \leq n)$. For QF_NIA formulas we can omit to consider strict inequalities as described in Table 1. This saves us from considering TCs with infinitesimals as introduced in 28 and the comparably higher complexity they entail. Therefore, $S\left(x_{j}\right)$ is either $-\infty$ or of the form $\frac{q_{j, 1}+q_{j, 2} \sqrt{q_{j, 3}}}{q_{j, 4}}$ with $q_{j, 1}, \ldots, q_{j, 4} \in \mathbb{Z}\left[x_{1}, \ldots, x_{j-1}\right]$ (roots parametrised in some polynomials).

If a solution $S$ for the relaxation $\varphi_{\mathbb{R}}^{n}$ is found then there is a true leaf in the search tree, as illustrated in Figure2. We now try to construct an integer solution $S^{*}$ from the parametrised solution $S$, as illustrated in Figure 4 traversing the solution path from the true leaf backwards. If the TC $t_{x_{1}}^{i_{1}}$ for $x_{1}$ is not $-\infty$, it does not contain any variables, thus we can determine whether its value is

\footnotetext{
${ }^{3}$ In the experimental results we use final lemmas only.

${ }^{4}$ Note that modern SAT solvers also allow to forget learnt clauses that did not contribute to conflicts recently. This applies also to branching clauses.
} 


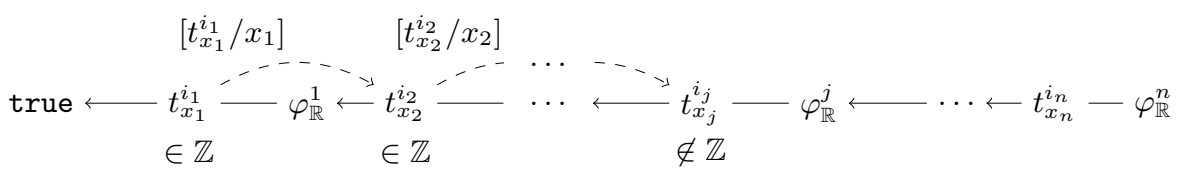

Fig. 4: Solution path from Figure 2 traversed backwards from the leaf to the root

integer and set $S^{*}\left(x_{1}\right)$ to this value. If $t_{x_{1}}^{i_{1}}=-\infty$, we can take any integer which is strictly smaller than all the other TCs in $T\left(x_{1}, \varphi_{\mathbb{R}}^{1}\right)$. Now we iterate backwards: for each test candidate $t_{x_{j}}^{i_{j}}$ on the solution path, which is not $-\infty$, we substitute the values $S^{*}\left(x_{1}\right), \ldots, S^{*}\left(x_{j-1}\right)$ for the variables $x_{1}, \ldots, x_{j-1}$, resulting in $S^{*}\left(x_{j}\right):=S\left(x_{j}\right)\left[S^{*}\left(x_{1}\right) / x_{1}\right] \ldots\left[S^{*}\left(x_{j-1}\right) / x_{j-1}\right]$, which again does not contain any variables and we can evaluate whether its value is integer. If $t_{x_{j}}^{i_{j}}=-\infty$ then we evaluate all test candidates from $T\left(x_{j}, \varphi_{\mathbb{R}}^{j}\right)$ whose side conditions hold by substituting $S^{*}\left(x_{1}\right), \ldots, S^{*}\left(x_{j-1}\right)$ for $x_{1}, \ldots, x_{j-1}$ in the TC expressions, and we set $S^{*}\left(x_{j}\right)$ to an integer value that is strictly smaller than all those TC values. We repeat this procedure until either a full integer solution is found or the resulting value is not integer in one dimension.

If all TC values are integer then VS returns sat. Otherwise, if we determine that $S^{*}\left(x_{j}\right)$ for some $j$ is not integer-valued, then there is some $z \in \mathbb{Z}$ such that $S^{*}\left(x_{j}\right) \in(z-1, z)$. In this case we return the branching lemma $\left(\bigwedge_{\psi \in \operatorname{Orig}_{x_{j}}\left(S\left(x_{j}\right)\right)} \psi\right) \Rightarrow\left(x_{j} \leq z-1 \vee x_{j} \geq z\right)$, where $\operatorname{Orig}_{x_{j}}\left(S\left(x_{j}\right)\right)$ denotes the VS module's received constraints being responsible for the creation of the TC $S\left(x_{j}\right)$. We can determine this set recursively with $\operatorname{Orig}_{x_{j}}\left(S\left(x_{j}\right)\right):=\operatorname{Orig}_{x_{j}}(c)$ if we used constraint $c \in \operatorname{Con}\left(\varphi_{\mathbb{R}}^{j}\right)$ for generating the TC $S\left(x_{j}\right)$, and where

$\operatorname{Orig}_{x_{j}}(c):= \begin{cases}c & , \text { if } j=n \\ \operatorname{Orig}_{x_{j+1}}(c) & , \text { if } x_{j} \notin \operatorname{Var}(c) \\ \operatorname{Orig}_{x_{j+1}}\left(S\left(x_{j+1}\right)\right) \cup \operatorname{Orig}_{x_{j+1}}\left(c^{\prime}\right), & \text { if } c^{\prime} \in \operatorname{Con}\left(\varphi_{\mathbb{R}}^{j+1}\right) \text { such that } \\ & c \in \operatorname{Con}\left(c^{\prime}\left[S\left(x_{j+1}\right) / / x_{j+1}\right]\right) \\ \operatorname{Orig}_{x_{j+1}}\left(S\left(x_{j+1}\right)\right) & , \text { otherwise. }\end{cases}$

Note that the last case occurs if the given constraint is introduced through a TC's side condition.

Basically, if we have found a non-integer valued TC $S^{*}\left(x_{j}\right) \notin \mathbb{Z}$, we can still continue the procedure to determine all other non-integer-valued TCs, but the gain (enabling a heuristics to select on which variable value we want to branch) comes at high computational costs, as we need to compute with nested fractions and square roots. Therefore, we do not consider other heuristics but branch always on the first detected non-integer value. In contrast, as we will see in the next section, the CAD methods offers more freedom to design other heuristics.

This procedure is sound, as we do not prune any integer solutions. It is not complete, as it might branch infinitely often for the same variable at an always 
increasing or always decreasing value. This procedure can also be used to check a QF_NIRA formula for satisfiability, if we eliminate real-valued variables first.

\section{Branch-and-Bound with the CAD Method}

Also the CAD method can be embedded into the BB approach the usual way, however, it offers more flexibility to tune its search towards integer solutions.

Sample point selection. The computation time of the lifting phase heavily depends on the representation size of the numbers involved. The representation of an integer is inherently smaller than that of a fractional number of a similar value due to the lack of a denominator and a smaller numerator. Therefore, when selecting a sample point from a given interval, we always choose an integer value whenever one exists. As a side effect, this is not only faster due to the smaller representation but also generates integer solutions automatically which generally helps to avoid unnecessary branches.

When several integer values are available in a given interval as possible sample points, some of them might lead to a full integer solution whereas some others not. Unfortunately, there is no generally valid rule to determine more "promising" samples. In our implementation we choose integer samples around the interval middle.

Note that we could even stop lifting for the given partial sample if a complete integer extension of the current sample becomes impossible during the lifting. However, if at an earlier level we had the choice between different integer values for a cell sample then we cannot conclude unsatisfiability in the current sub-tree. Therefore, even if we cannot choose integer samples, in the current implementation we continue lifting and search for a satisfying real extension of the partial integer sample. If the search leads to a (non-integer) solution, we request branching at the SAT level. Otherwise, if the current branch is unsatisfiable in the real domain, we continue the search in other parts of the search space.

Example 1. Consider $P_{2}=\left\{x_{2}^{2}+x_{1}=0, x_{1}<-1\right\}$. Projecting $x_{2}$ yields $P_{1}=$ $\left\{x_{1}, x_{1}+1\right\}$ with real roots $\{-1,0\}$. To satisfy $x_{1}<-1$, we only need to choose a sample for $x_{1}$ from $(-\infty,-1)$. Assume we choose -2 . Lifting -2 yields the polynomials $\left\{x_{2}^{2}-2,-1\right\}$ with real roots $\{-\sqrt{2}, \sqrt{2}\}$, both being non-integer. All other cells around these roots violate the sign condition. However, we cannot infer unsatisfiability in the integer domain: selecting -4 instead of -2 would have produced the polynomials $\left\{x_{2}^{2}+4,-3\right\}$ with integer roots $\{-2,2\}$.

Remark 1. We would like to mention an idea, which is not yet implemented but might lead to further improvements. Assume as above an integer sample $s=\left(s_{1}, \ldots, s_{j}\right) \in \mathbb{Z}^{j}$ for which lifting yields no integer extension. Assume now additionally that in each dimension $i=1, \ldots, j$ the sample $s_{i}$ is the only integer point in the respective interval; we say that $s$ is unique. In this special case the current sub-tree cannot contain any integer solutions; we can safely stop lifting for $s$ and continue in other parts of the search space. 
If we find a solution elsewhere, we can return sat. However, if the input formula has no integer solution, CAD needs to return an explanation for unsatisfiability. In the real domain, we generate such an explanation by specifying for each full-dimensional sample $s$ (i.e., for each leaf in the lifting tree) the set $E_{s}$ of all original constraints that are violated by the leaf, and computing a possibly small covering set $E$ which contains at least one constraint from each leaf's set $E_{s}$.

Now, if we do not complete the lifting for some sub-trees because we determined unsatisfiability at an earlier level, we cannot use the same approach to generate explanations. Instead, we can proceed as follows: Remember that $s=\left(s_{1}, \ldots, s_{j}\right) \in \mathbb{Z}^{j}$ is the sample for which lifting was stopped because $s$ is unique and it has no integer extension. Each $s_{i}$ samples an interval, whose endpoints are zeros of some polynomials from $P_{i}$ at $\left(s_{1}, \ldots, s_{i-1}\right)$; let $P_{i}^{s} \subseteq P_{i}$ be the set of those polynomials for $i=1, \ldots, j$ and let $P_{i}^{s}=\emptyset$ for $i=j+1, \ldots, n$. Now we follow back the projection tree, and for $i=1, \ldots, n-1$ we iteratively add to $P_{i+1}^{s}$ all "projection parents" ${ }^{5}$ of all polynomials in $P_{i}^{s}$, i.e., all those polynomials that were used in the projection to generate $P_{i}^{s}$. As a result we achieve a set $P_{n}^{s} \subseteq P_{n}$ of original constraints, which serve as an explanation for the unsatisfiability of the sub-tree rooted at $s$. We compute this set $P_{n}^{s}$ for each unique non-completed sample, build their union, and further extend it with additional constraints from $P_{n}$ to cover all sets $E_{s^{\prime}}$ of full-dimensional sample leafs $s^{\prime}$. The resulting set is an infeasible subset of the input constraint set.

Remark 2. As the selection of sample points might be crucial for discovering integer solutions, we also experimented with choosing (if possible) multiple sample points for an interval instead of a single one. However, the overhead due to these redundant sample points greatly outweighs any gain, even if only two samples for a single interval are chosen. This is because the redundancy increases with every dimension and often leads to an additional exponential growth.

Sample point lifting order. The order in which sample points are lifted is crucial for fast solution finding. As we want to find integer solutions, we first lift integer sample points before considering non-integer ones. Furthermore, if we already have a partial lifting tree due to a previous incremental call to the CAD method, for further lifting we choose partial integer sample points of high dimension first.

Constructing branching lemmas. If CAD finds a solution $s=\left(s_{1}, \ldots, s_{n}\right) \in \mathbb{R}^{n} \backslash \mathbb{Z}^{n}$, it returns unknown and requests branching at the SAT level. We have tried three alternative strategies to generate branching lemmas. The first strategy branches on the value of $x_{i}$ with $i=\min \left\{i=1, \ldots, n \mid s_{i} \notin \mathbb{Z}\right\}$. The second strategy is similar but takes the highest index. In both cases, the branching premise is the set of all received constraints; in the future we will also experiment with the set $P_{n}^{s}$ (see Remark 1).

The third strategy makes use of the sampling heuristics of the CAD that strongly prefers integers. That means that the longest integer prefix $\left(s_{1}, \ldots, s_{j}\right) \in$

\footnotetext{
${ }^{5}$ A projected polynomial can have several "parents"; in this case we can choose any of them. In practice, one could store the chronologically "oldest" parents, as backtracking removes input polynomials in chronologically reverse order.
} 
$\mathbb{Z}^{j}$ of $s$ cannot be further extended with an integer sample component. This strategy generates the branching lemma

$$
C \rightarrow\left(\bigvee_{i=1}^{j} x_{i} \leq s_{i}-1 \vee x_{i} \geq s_{i}+1\right)
$$

Currently, the branching premise $C$ is again the set of all received constraints. In the future we will also investigate collecting all constraints that reject integer sample points in the vicinity of the first non-integer component $s_{j+1}$. Let $s_{j+1 \downarrow}$ $\left(s_{j+1 \uparrow}\right)$ be $s$ where $s_{j+1}$ is replaced by $\left\lfloor s_{j+1}\right\rfloor\left(\left\lceil s_{j+1}\right\rceil\right)$. We define the branching premise by $\left\{c \in C \mid c\left(s_{j+1 \downarrow}\right) \equiv\right.$ false $\vee c\left(s_{j+1 \uparrow}\right) \equiv$ false $\}$.

\section{Combination of Procedures}

We can often improve the performance for solving QF_NRA formulas if we have different decision procedures at hand and use them in combination such that theory modules can pass on sub-problems for satisfiability check to other modules. In SMT-RAT, such combinations of decision procedures were already available for QF_NRA problems. In this section we discuss how to extend the approach for QF_NIA and the BB framework, on the examples of theory modules implementing the simplex, VS and CAD methods.

Given a set $C$ of non-linear integer arithmetic constraints, the simplex method can be used to check the consistency of the relaxed linear constraints in $C$, first neglecting the non-linear ones. If simplex determines that the real relaxation of the linear part of the problem is unsatisfiable, it returns unsat. If it finds an integer solution that also satisfies the non-linear constraints, it returns sat. If it finds a solution that is not completely integer, but satisfies the real relaxation of the non-linear constraints, it creates a branching lemma and returns unknown. Otherwise, it forwards the whole input $C$ to another theory solving module, and passes back the result and, if constructed, the branching lemma to its caller.

For VS, assume that we eliminate the variable $x_{j}(1 \leq j \leq n)$ from the formula $\varphi_{\mathbb{R}}^{j}$ as illustrated in Figure 2. In general, we can also use the virtual substitution if in some of the polynomials in $\varphi_{\mathbb{R}}^{j}$ the degree of $x_{i}$ is higher than 2 : We generate all test candidates for $x_{j}$ from all constraints in which $x_{j}$ appears at most quadratic. If any of those test candidates leads to a satisfying solution, we can conclude the satisfiability of $\varphi_{\mathbb{R}}^{j}$. Otherwise, we can pass the sub-problem $\varphi_{\mathbb{R}}^{j}$ to another theory solving module for satisfiability check. If it returns unsat, we have to consider another path in the search tree of Figure 2 If it returns sat, we can use the integer assignment of the variables in the passed sub-problem to construct an integer solution for the remaining variables as explained in Section 4 Finally, if the sub-call returns unknown and constructs a branching lemma, then VS returns unknown and passes the branching lemma through. If there is no other theory solving module to be called, the sub-call also returns unknown.

\footnotetext{
${ }^{6}$ This form of multiple-branch lemmas are handled analogously to the 2-branch-case.
} 
The CAD theory solving module implements a complete decision procedure for QF_NRA. For this logic, the CAD module does not pass on any sub-problems to other solver modules.

\section{$7 \quad$ Experimental Results}

We consider a sequential strategy a sequence of modules that call each other sequentially as described before and we denote it by $\mathrm{M}_{1} \rightarrow \ldots \rightarrow \mathrm{M}_{k}$ where $\mathrm{M}_{1}$ may issue sub-calls to $\mathrm{M}_{2}$ and so on. We evaluated different sequential strategies for solving QF_NIA formulas, using the following modules $M_{i}$ :

- The SAT solver module $\mathrm{M}_{\mathrm{SAT}}$ behaves as explained in Section 3

- MSATStop works similarly except that it returns unknown if an invoked theory solver module returns unknown, instead of continuing the search for further Boolean assignments. The module $\mathrm{M}_{\mathrm{SAT}}$ stop provides us a reference: if this module is able to solve a problem then the problem can be considered irrelevant for BB (as BB was not involved).

- The module $M_{L R A}$ implements the simplex method with branching lemma generation, as explained in Section 6 .

- The theory solver modules $\mathrm{M}_{\mathrm{VS}}$ (implementing VS) and $\mathrm{M}_{\mathrm{CAD}}$ (implementing CAD) check the real relaxation of a QF_NIA input formula. If the relaxation is unsatisfiable they return unsat, if they coincidentally find an integer solution they return sat, otherwise they return unknown (without applying BB).

- The VS module $\mathrm{M}_{\mathrm{VS}_{\mathbb{Z}}}$ constructs branching lemmas as explained in Section 4 .

- The CAD modules $\mathrm{M}_{\mathrm{CAD}} \mathrm{F}_{\mathbb{Z}}^{\mathrm{First}}$ and $\mathrm{M}_{\mathrm{CAD}}^{\mathrm{Last}}$ construct branching lemmas (Sec. 5 ) based on the first- resp. last-lifted variable with a non-integer assignment.

- The CAD module $\mathrm{M}_{\mathrm{CAD}}$ Path constructs branching lemmas which exclude the longest integer prefix of the found non-integer solutions (Eq. 3).

- Bit-blasting is implemented in the module $\mathrm{M}_{\text {IntBlast }}$. In our strategies it will be combined with a preceding incremental variable bound widening module $\mathrm{M}_{\text {IncWidth }}$, which constrains, for instance, first that all variables are in $[-1,2]$, if no solution can be found, it requires all variables to be in $[-3,4]$ etc.

All experiments were carried out on AMD Opteron 6172 processors. Every solver was allowed to use up to 4 GB of memory and 200 seconds of wall clock time.

For our experiments we used the largest benchmark sets for QF_NIA from the last SMT-COMP: APRove, LEIPZIG (both generated by automated termination analysis) and CALYPTO (generated by sequential equivalence checking). Additionally, we crafted a new benchmark set $\mathrm{CALYPTO}_{\infty}$ by removing all variable bound constraints from CALYPTO and thereby obtaining unbounded problems (together 8572 problem instances, see headline in Fig. 5e for the size of each set). Selection of a VS heuristic The SMT-RAT strategy $\mathrm{M}_{\mathrm{SAT}_{\mathrm{Stop}}} \rightarrow \mathrm{M}_{\mathrm{VS}}$ could solve 7215 sat and 84 unsat instances, ran out of time or memory for 1146 instances, and returned unknown for 127 instances. Applying the SMT-RAT strategy $\mathrm{M}_{\mathrm{SAT}} \rightarrow \mathrm{M}_{\mathrm{VS}}$ to those 127 instances, we can solve an additional 30 sat instances. If 


$$
\begin{array}{ll}
\mathrm{RAT}_{\mathbb{Z}}: & \mathrm{M}_{\mathrm{SAT}} \longrightarrow \mathrm{M}_{\text {LRA }} \longrightarrow \mathrm{MVS}_{\mathbb{Z}} \longrightarrow \mathrm{M}_{\mathrm{CAD}} \mathrm{F}_{\mathbb{Z}} \text { irst } \\
\mathrm{RAT}_{\text {blast }}: & \mathrm{M}_{\text {IncWidth }} \longrightarrow \mathrm{M}_{\text {IntBlast }} \\
\mathrm{RAT}_{\text {blast. }}: & \mathrm{M}_{\text {IncWidth }} \longrightarrow \mathrm{M}_{\text {IntBlast }} \longrightarrow \mathrm{RAT}_{\mathbb{Z}}
\end{array}
$$

(a) The SMT-RAT strategies [14] used for the experimental results

\begin{tabular}{l||rr|rr|} 
& & $\mathrm{VS}_{\mathbb{R}}$ & \multicolumn{3}{c|}{$\mathrm{VS}_{\mathbb{Z}}$} \\
& $\#$ & time & $\#$ & time \\
\hline \hline sat & 30 & 714.2 & $\mathbf{9 3}$ & $\mathbf{4 8 7 . 3}$ \\
unsat & 0 & 0.0 & $\mathbf{1 0}$ & $\mathbf{9 . 2}$
\end{tabular}

(b) Comparison of 2 VS heuristics on 126

\begin{tabular}{|c|c|c|c|c|c|c|c|c|}
\hline & & $\begin{array}{c}\mathrm{AD}_{\mathbb{R}} \\
\text { time }\end{array}$ & $\begin{array}{l}\mathrm{CA} \\
\#\end{array}$ & $\begin{array}{c}\mathrm{D}_{\mathbb{Z}}^{\text {First }} \\
\text { time }\end{array}$ & $\begin{array}{l}\mathrm{CA} \\
\#\end{array}$ & $\begin{array}{c}\mathrm{D}_{\mathbb{Z}}^{\text {Last }} \\
\text { time }\end{array}$ & & $\begin{array}{l}\mathrm{D}_{\mathbb{Z}}^{\text {Path }} \\
\text { time }\end{array}$ \\
\hline & 12 & 137.7 & 12 & 183.5 & 11 & 182.7 & . & 88. \\
\hline isat & 0 & 0.0 & 13 & 150.8 & 13 & 151.0 & 2 & 131.9 \\
\hline
\end{tabular}
(101 sat, 25 unsat) for $\mathrm{BB}$ relevant instances

(c) Comparison of $4 \mathrm{CAD}$ heuristics on 55

(27 sat, 28 unsat) for BB relevant instances

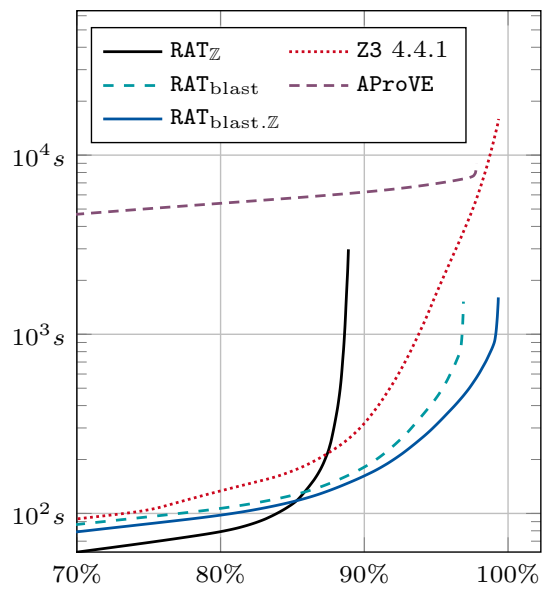

\begin{tabular}{|c|c|c|c|c|c|c|c|c|c|c|c|}
\hline \multicolumn{2}{|c|}{$\begin{array}{l}\text { Benchmark } \rightarrow \\
\text { Solver } \downarrow\end{array}$} & \multicolumn{2}{|c|}{$\begin{array}{c}\text { AProve }(8129) \\
\# \quad \text { time }\end{array}$} & \multicolumn{2}{|c|}{ Calypto (138) } & \multicolumn{2}{|c|}{ LEIPZIG (167) } & \multicolumn{2}{|c|}{ CALYPTO $_{\infty}(138)$} & $\begin{array}{r}\text { all } \\
\#\end{array}$ & $\begin{array}{r}(8572) \\
\text { time }\end{array}$ \\
\hline \multirow[t]{2}{*}{$\overline{\operatorname{RAT}_{\mathbb{Z}}}$} & sat & 7283 & 2294.8 & 67 & 71.2 & 9 & 260.4 & 133 & 298.9 & 7492 & 2925.3 \\
\hline & & 73 & 14.3 & 52 & 40.7 & 0 & 0.0 & 3 & $<0.1$ & 128 & 55.1 \\
\hline \multirow{2}{*}{$\overline{\text { RAT }}_{\text {blast }}$} & $\mathrm{S}$ & 8025 & 866.3 & 21 & 35.6 & 156 & 603.3 & 87 & 16.0 & 8289 & 1521.2 \\
\hline & $\mathrm{u}$ & 12 & 0.4 & 5 & 0.1 & 0 & 0.0 & 0 & 0.0 & 17 & 0.5 \\
\hline \multirow[t]{2}{*}{$\overline{\mathrm{RAT}_{\text {blast } . \mathbb{Z}}}$} & sat & 8025 & 780.7 & 79 & 122.3 & 156 & 511.5 & 134 & 21.8 & 8394 & 1436.3 \\
\hline & unsat & 71 & 42.6 & 46 & 127.5 & 0 & 0.0 & 3 & 0.1 & 120 & 170.2 \\
\hline \multirow[t]{2}{*}{$\overline{\mathrm{Z3}}$} & sat & 7992 & 14695.5 & 78 & 19.1 & 158 & 427.6 & 126 & 57.3 & 8354 & 15199.5 \\
\hline & unsat & 102 & 595.9 & 57 & 117.6 & 0 & 0.0 & 3 & 2.3 & 162 & 715.8 \\
\hline \multirow[t]{2}{*}{$\overline{\text { AProVE }}$} & $\mathrm{sc}$ & 8025 & 7052.2 & 74 & 559.1 & 159 & 696.5 & 127 & 685.2 & 8385 & 8993.0 \\
\hline & unsat & 0 & 0.0 & 0 & 0.0 & 0 & 0.0 & 0 & 0.0 & 0 & 0.0 \\
\hline
\end{tabular}

(d) Cumulative time to solve instances from all benchmark sets

(e) Comparison of 3 SMT-RAT strategies to currently fastest SMT solvers for QF_NIA

Fig. 5: The column \# contains the number of solved instances and the column time contains the amount of seconds needed for solving these instances

we replace the module $\mathrm{M}_{\mathrm{VS}}$ by the $\mathrm{M}_{\mathrm{VS}_{\mathbb{Z}}}$ module, which applies branching lemmas, we can solve further 63 sat and 10 unsat instances (see Figure 5b.

Selection of a CAD heuristic The SMT-RAT strategy $\mathrm{M}_{\mathrm{SAT}_{\mathrm{Stop}}} \rightarrow \mathrm{M}_{\mathrm{CAD}}$ could solve 6656 sat and 26 unsat instances. The main reason why this approach can already solve more than $77 \%$ of the examples lies in the nature of the CAD to choose preferably integer sample points and, of course, in the structure of the benchmark instances. The strategy ran out of time or memory for 1835 instances, and returned unknown for 55 instances. On these 55 examples, we compared the SMT-RAT strategy $\mathrm{M}_{\mathrm{SAT}} \rightarrow \mathrm{M}_{\mathrm{CAD}}$ and 3 other strategies replacing the $\mathrm{M}_{\mathrm{CAD}}$ module by $\mathrm{M}_{\mathrm{CAD}}^{\text {First }}, \mathrm{M}_{\mathrm{CAD}}^{\text {Last }}$ and $\mathrm{M}_{\mathrm{CAD}} \mathrm{D}_{\mathbb{Z}}^{\text {Path }}$. As shown in Table 5c we find 12 additional sat instances with the $\mathrm{M}_{\mathrm{CAD}}$ module. The BB modules $\mathrm{M}_{C A D}$ First and $\mathrm{M}_{\mathrm{CAD}} \mathrm{Z}_{\mathbb{Z}}^{\text {Last }}$ perform very similar and find 13 additional unsat instances. This is due 
to the fact that almost always the assignment of only one variable was not yet integer. With the heuristic in the module $\mathrm{M}_{C A D_{\mathrm{Z}}^{\text {Path }}}$ we could solve less instances. Combined strategies We crafted three strategies, depicted in Fig. 5a, to combine different theory solver modules 7 . The strategy RAT $\mathrm{T}_{\text {blast. }} \mathbb{Z}$ combines $\mathrm{RAT}_{\text {blast }}$ and $\mathrm{RAT}_{\mathbb{Z}}$ by first using bit-blasting up to a width of 4 bits. If this does not yield a solution, it continues to use $\mathrm{RAT}_{\mathbb{Z}}$.

We compared these three strategies with the two fastest SMT solvers from the 2015 SMT-COMP for QF_NIA: Z3 and AProVE. Though CVC4 performed worse than these two solvers, its experimental version solved slightly more instances than AProVE in about half of the time; we did not include it here but expect it to perform between $\mathrm{Z3}$ and $\mathrm{AProVE}$. Figure $5 \mathrm{e}$ shows that $\mathrm{RAT}_{\mathbb{Z}}$ and $\mathrm{RAT}_{\text {blast }}$ complement each other well, especially for satisfiable instances. Compared to Z3 and AProVE, RAT blast.Z solves more satisfiable instances and does this even faster by a factor of more than 10 and 6 , respectively. The strategy $\mathrm{RAT}_{\mathbb{Z}}$ solves less instances, but, as shown in Figure 5d, this strategy solves the first 85 percent of the examples faster than any other SMT-RAT strategy or SMT solver. On unsatisfiable instances, however, Z3 is still better than SMT-RAT while AProVE is not able to deduce unsatisfiability due to its pure bit-blasting approach.

We also tested all SMT-RAT strategies which use BB, once with and once without using a branching premise. Here we could not detect any notable difference, which we mainly relate to the fact that those problem instances, for which BB comes to application, are almost always pure conjunctions of constraints and involve only a small number of branching lemma liftings. For a more reliable evaluation a larger set of QF_NIA benchmarks would be needed.

\section{Conclusion and Future Work}

The efficiency of solving QF_NIA formulas highly depends on a good strategic combination of different procedures. In this paper we comprised two algebraic procedures, the virtual substitution and the cylindrical algebraic decomposition methods, in a combination with the branch-and-bound approach, which has already been applied effectively in combination with the simplex method. We showed by experimental evaluation that this combination highly complements bit-blasting, the currently most efficient approach for QF_NIA.

The next steps to enhance the strategy for solving QF_NIA formulas could involve interval constraint propagation in order to infer better bounds for the variables. We also plan to further optimise the generation of branching lemmas and of the explanations of unsatisfiability in the theory solving modules.

\section{References}

1. Arnon, D.S., Collins, G.E., McCallum, S.: Cylindrical Algebraic Decomposition I: The Basic Algorithm. SIAM Journal on Computing 13(4), 865-877 (1984)

\footnotetext{
7 Additionally, all of these strategies employ a common preprocessing.
} 
2. Arnon, D.S., Collins, G.E., McCallum, S.: Cylindrical Algebraic Decomposition II: An Adjacency Algorithm for the Plane. SIAM Journal on Computing 13(4), 878-889 (1984)

3. Barnett, M., Chang, B.Y.E., DeLine, R., Jacobs, B., Leino, K.R.M.: Proc. of FMCO'05, chap. Boogie: A Modular Reusable Verifier for Object-Oriented Programs, pp. 364-387. Springer (2006)

4. Barrett, C., Conway, C.L., Deters, M., Hadarean, L., Jovanović, D., King, T., Reynolds, A., Tinelli, C.: CVC4. In: Proc. of CAV'11. vol. 6806, pp. 171-177. Springer (2011)

5. Barrett, C., Nieuwenhuis, R., Oliveras, A., Tinelli, C.: Splitting on demand in SAT modulo theories. In: Proc. of LPAR'06. vol. 4246, pp. 512-526. Springer (2006)

6. Barrett, C., Sebastiani, R., Seshia, S.A., Tinelli, C.: Satisfiability modulo theories. In: Handbook of Satisfiability, Frontiers in Artificial Intelligence and Applications, vol. 185, chap. 26, pp. 825-885. IOS Press (2009)

7. Biere, A., Heule, M.J.H., van Maaren, H., Walsh, T. (eds.): Handbook of Satisfiability, Frontiers in Artificial Intelligence and Applications, vol. 185. IOS Press (2009)

8. Borralleras, C., Lucas, S., Navarro-Marset, R., Rodríguez-Carbonell, E., Rubio, A.: Solving Non-linear Polynomial Arithmetic via SAT Modulo Linear Arithmetic. In: Proc. of CADE-22, vol. 5663, pp. 294-305. Springer (2009)

9. Bouton, T., de Oliveira, D.C.B., Déharbe, D., Fontaine, P.: veriT: An open, trustable and efficient SMT-solver. In: Proc. of CADE-22. vol. 5663, pp. 151-156. Springer (2009)

10. Brown, C.W.: Improved Projection for Cylindrical Algebraic Decomposition. Journal of Symbolic Computation 32(5), 447 - 465 (2001)

11. Cimatti, A., Griggio, A., Schaafsma, B., Sebastiani, R.: The MathSAT5 SMT solver. In: Proc. TACAS'13, vol. 7795, pp. 93-107. Springer (2013)

12. Collins, G.E.: Quantifier elimination for real closed fields by cylindrical algebraic decomposition. In: Automata Theory and Formal Languages. vol. 33, pp. 134-183. Springer (1975)

13. Corzilius, F., Ábrahám, E.: Virtual substitution for SMT solving. In 18th Int. Symp. on Fundamentals of Computation Theory (2011)

14. Corzilius, F., Kremer, G., Junges, S., Schupp, S., Ábrahám, E.: SMT-RAT: An open source C++ toolbox for strategic and parallel SMT solving. In: Proc. of SAT'15. vol. 9340, pp. 360-368. Springer (2015)

15. Dantzig, G.B.: Linear programming and extensions. Princeton University Press (1963)

16. Davis, M., Logemann, G., Loveland, D.: A machine program for theorem-proving. Commun. ACM 5(7), 394-397 (1962)

17. Dutertre, B., de Moura, L.M.: A fast linear-arithmetic solver for DPLL(T). In: Proc. of CAV'06. vol. 4144, pp. 81-94. Springer (2006)

18. Dutertre, B.: Yices 2.2. In: Proc. of CAV'14. vol. 8559, pp. 737-744. Springer (2014)

19. Fränzle, M., Herde, C., Teige, T., Ratschan, S., Schubert, T.: Efficient solving of large non-linear arithmetik constraint systems with complex Boolean structure. Journal on Satisfiability, Boolean Modeling and Computation 1, 209-236 (2007)

20. Fuhs, C., Giesl, J., Middeldorp, A., Schneider-Kamp, P., Thiemann, R., Zankl, H.: SAT solving for termination analysis with polynomial interpretations. In: Proc. of SAT'07. vol. 4501, pp. 340-354. Springer, Lisbon (2007)

21. Griggio, A.: A practical approach to satisfiability modulo linear integer arithmetic. Journal on Satisfiability, Boolean Modeling and Computation 8, 1-27 (2012) 
22. Hong, H.: An Improvement of the Projection Operator in Cylindrical Algebraic Decomposition. In: Proc. of ISSAC'90. pp. 261-264. ACM (1990)

23. Khanh, T.V., Vu, X., Ogawa, M.: raSAT: SMT for Polynomial Inequality. In: Proc. of SMT'14. p. 67 (2014)

24. Kim, H., Somenzi, F., Jin, H.: Efficient term-ITE conversion for satisfiability modulo theories. In: Proc. of SAT'09. vol. 5584, pp. 195-208. Springer (2009)

25. McCallum, S.: An Improved Projection Operation for Cylindrical Algebraic Decomposition of Three-dimensional Space. Journal of Symbolic Computation 5(1), $141-161(1988)$

26. de Moura, L.M., Bjørner, N.: Z3: An efficient SMT solver. In: Proc. of TACAS'08. vol. 4963, pp. 337-340. Springer (2008)

27. Schrijver, A.: Theory of Linear and Integer Programming. Wiley (1986)

28. Weispfenning, V.: Quantifier elimination for real algebra - the quadratic case and beyond. Appl. Algebra Eng. Commun. Comput. 8(2), 85-101 (1997) 